\title{
Pastorale missionnaire de l'abbé Hyppolite Beillevaire
}

\section{Éméric Drouin}

Volume 37, 1970

La vie religieuse au Manitoba

URI : https://id.erudit.org/iderudit/1007283ar

DOI : https://doi.org/10.7202/1007283ar

Aller au sommaire du numéro

Éditeur(s)

Les Éditions Historia Ecclesiæ Catholicæ Canadensis Inc.

ISSN

0318-6172 (imprimé)

1927-7067 (numérique)

Découvrir la revue

Citer cet article

Drouin, É. (1970). Pastorale missionnaire de l'abbé Hyppolite Beillevaire.

Sessions d'étude - Société canadienne d'histoire de l'Église catholique, 37,

197-213. https://doi.org/10.7202/1007283ar

Tous droits réservés @ Les Éditions Historia Ecclesiæ Catholicæ Canadensis Inc., 1972
Ce document est protégé par la loi sur le droit d'auteur. L'utilisation des services d'Érudit (y compris la reproduction) est assujettie à sa politique d'utilisation que vous pouvez consulter en ligne.

https://apropos.erudit.org/fr/usagers/politique-dutilisation/ 


\section{Pastorale missionnaire de l'abbé Hyppolite Beillevaire}

Il est difficile de classifier la forme de pastorale employée par les missionnaires depuis la fondation du premier poste sur le territoire albertain, celui du Lac Sainte-Anne, jadis connu sous le nom du Lac Machimanitou - Lac du Diable - , fondation de l'abbé Thibeau en 1842 durant sa seconde visite aux Indiens des Plaines, jusqu'à la disparition presque totale, en 1877, des millions de buffalos. Ce prêtre vint surtout sur l'insistance de Piché, Canadien français marié à une Indienne et devenu chef d'une tribu crise dont les descendants demeurent à Hobbéma, à l'endroit même où l'abbé Beillevaire a son premier pied-à-terre.

Peu de missions stables existent, surtout par manque de personnel. Le travail est plus suivi et quelque peu plus en profondeur aux environs des missions permanentes, mais ce sont surtout les Métis qui en profitent. Avant 1871, année où $\mathrm{M}^{\mathrm{gr}}$ Grandin s'installe à Saint-Albert à partir de l'île-à-la-Crosse, les efforts produisent peu de résultats chez les Indiens tandis que chez les Métis c'est plus consolant bien que les saints canonisables parmi eux ne foisonnent pas non plus.

La signature du Traité \# 6 entre les Cris et le gouvernement fédéral aux Forts Pitt et Carleton en 1876, et du Traité \# 7 à Blackfoot Crossing, en 1877, avec la confédération du sud, est le point de départ d'une nouvelle ère pastorale sans que, toutefois, le travail religieux devienne bien facile. La délimitation de réserves débute alors. Il se passe des années avant que les indigènes veuillent finalement être parqués dans ces territoires restreints établis non pas, comme les gens du gouvernement et certains historiens veulent qu'on le croit, pour protéger les autochtones contre les Blancs envahisseurs des Plaines, mais bien plutôt pour enlever les Indiens de territoires convoités par ces nouveaux arrivants. Un événement tragique pour les indigènes, la disparition quasi subite du buffalo, hâte l'application de cette politique, car les gens deviennent des crève-faim que nombre d'agents gouvernementaux dédaignent souverainement mais auxquels ils font une multitude de vaines promesses. Il faudra pressions sur pressions par les missionnaires de toutes les sectes et d'autres amis des Indiens; il faudra même des démonstrations belliqueuses de ces Indiens eux-mêmes pour qu'Ottawa s'émeuve un peu de la situation inhumaine. Plutôt par intérêt stomacal les tribus demandent des missionnaires résidents parmi elles, mais cela ne produit pas un mouvement de conversion massive et sincère. Les missionnaires, dès lors, étant plus constamment sur place peuvent et 
doivent appliquer les principes de la pastorale d'évangélisation. En effet, la plupart sont admirables de patience, d'esprit de sacrifice, de longanimité avec les pauvres hères, frappant sans cesse à leur porte, campant autour des missions ou même à l'intérieur de leurs huttes où existe habituellement une salle ad hoc, quémandant toujours, ou encore durant leurs visites répétées à domicile dans le but de soigner les malades avec des moyens par trop rudimentaires, de baptiser les enfants, d'instruire le peu d'adultes qui enfin consentent à écouter plus attentivement. Les voyages multiples se font ou à pied ou à dos de cheval par toutes les saisons et tous les temps. Cet apostolat, au lieu de devenir plus facile avec les années, devient plus difficile lorsque le gouvernement, voulant faire des fermiers des Indiens, les forcent à abandonner leurs camps plus ou moins populeux établis à proximité de rivières, de lacs et de forêts, pour se construire des masures un peu partout sur la prairie.

Même un homme aussi surnaturel que $\mathrm{M}^{\mathrm{gr}}$ Grandin, tellement découragé des minces résultats d'efforts héroïques de ses Pères et Frères dans le sud, décide, à deux reprises, de les retirer pour s'en servir ailleurs où la population les accueillerait avec reconnaissance. Ce n'est qu'à l'insistance des missionnaires eux-mêmes, espérant malgré tout, qu'il les laisse sur place.

Sans le travail bénévole des Frères convers et le dévouement des Sœurs Grises, soit de Montréal soit de Nicolet, pour les dispensaires et pour les petites écoles, les résultats apostoliques auraient été encore plus piètres qu'ils ne le sont alors.

Durant le dernier quart du XIX ${ }^{\bullet}$ siècle, quelques missions indiennes et blanches s'ajoutent aux autres établies auparavant. Dans les paroisses blanches on vise surtout à la pastorale de chrétienté, mais les curés y sont intimement mêlés aux problèmes sociaux, éducationnels, familiaux et même économiques. En général, ils demeurent plutôt longtemps aux mêmes postes, ils visitent constamment les familles, ils catéchisent dans les écoles, ils instaurent les dévotions et les sociétés habituelles dans le but d'intérioriser la foi chez leurs ouailles. Celles-ci, en général, ont grand respect pour leur clergé et pour les religieuses, là où il en existent, mais elles ne sont pas du tout gênées avec eux et elles. Le piédestal sur lequel on les pose n'est pas tellement élevé ! C'est plutôt l'atmosphère de paroisses de campagne qui prévaut. Le curé est un père de famille pour tous, ou à peu près, un père facilement consulté mais pas toujours écouté, un père même critiqué.

La plupart des paroisses alors sont de langue française, car l'élément anglophone catholique est plutôt restreint. Cette situation change toutefois avec l'immigration massive un peu avant mais surtout après la Première Guerre mondiale. 
Considérons maintenant l'œuvre d'un seul homme, de l'abbé Hyppolite Beillevaire, à Duhamel, où il emploie les deux sortes de pastorale, celle d'évangélisation et celle de chrétienté. La première surtout tant que les Blancs ne chassent pas ses Métis.

Beillevaire, né en 1848 à Sainte-Pazanne, Loire-Atlantique, à quelques milles au sud de Nantes, bien que prêtre séculier depuis quatre ans, est recruté en 1879 par $\mathbf{M}^{\mathrm{gr}}$ Vital Grandin, o.m.i., pour les Territoires du Nord-Ouest. Durant le même voyage en France, l'évêque persuade un professeur de mathématiques et de science de s'adjoindre au groupe qui viendra bientôt. Il s'agit de l'abbé Émile Legal, plus tard oblat, coadjuteur et successeur du premier évêque de Saint-Albert. L'embarquement est brusqué par suite des Lois Ferry qui chassent bien des religieux de leurs demeures, et Émile Legal est alors, ou en 1880, au noviciat.

Tout le personnel sous la houlette de $\mathrm{M}^{\mathrm{gr}}$ Grandin étant oblat, Beillevaire commence son noviciat à Saint-Albert en septembre 1880, mais au printemps de 1881 il le discontinue pour deux raisons: la première est qu'on en a absolument besoin pour fonder une mission à la Montagne de l'Ours, ou Notre-Dame-des-Sept-Douleurs, plus tard appelée Hobbéma; la seconde est que l'évêque ne le trouve pas assez mortifié lorsqu'il le découvre en train de se faire rôtir une perdrix en dehors du temps des repas. Ajoutons immédiatement toutefois que l'abbé, de fait, aura une vie tout aussi et même plus difficile que celle de n'importe quel autre missionnaire, car il demeure seul toujours.

Parti de Saint-Albert le 27 avril 1881 avec $M^{\text {gr }}$ Grandin, deux Frères et un jeune Métis qui doit demeurer avec lui, Beillevaire met onze jours à couvrir soixante-cinq milles. Les chemins et la température sont abominables.

En hâte, parce que l'évêque et ses compagnons doivent visiter les missions $d u$ sud et parce que les vivres font défaut ${ }^{1}$, on monte une partie de la masure que doivent habiter le jeune prêtre et son homme. On lui laisse la responsabilité de la finir, mais ni lui ni son Métis ne semblent connaître quoi que ce soit de l'art du charpentier, de sorte que le toit, mal posé, sera bientôt emporté par le vent. Parfois, il essaye de vivre dans cette cambuse, parfois il demeure chez le chef Peau-deBelette (Erminskin), descendant polygame de l'ancien chef Piché.

A l'automne, craignant les rigueurs de l'hiver et plutôt découragé par le peu de succès de son ministère auprès d'une population surtout préoccupée de lui quêter ses vivres, Beillevaire se transporte à environ

1 Pour détails sur cette fondation, voir les biographies de $\mathrm{M}^{\mathrm{gr}}$ Grandin, la brochure Hobbéma du père P.-E. BREToN, 0.m.i., et, en traduction, Hobbéma, Ongoing Indian Mission of Central Alberta, par E. O. Drouin, o.m.i.; ainsi que les rapports de $\mathrm{M}^{\mathrm{gr}}$ Grandin et de $\mathrm{H}$. Beillevaire aux archives oblates, Edmonton. 
vingt-six milles plus à l'est à la Rivière Bataille, plus tard appelée Salois ou Laboucane Settlement et enfin Duhamel. Dès lors et jusqu'à son décès en 1937, c'est son lieu de domicile. De là il revient parfois visiter les Indiens d'Hobbéma jusqu'à l'arrivée d'Oblats en 1884, et de là il rayonne dans la plaine pour apporter les réconforts religieux à de petits groupes de catholiques disséminés ici et là. Sa réputation, quasi légendaire, $y$ est encore vivave.

Il demeure un temps chez Elzéar Laboucane ${ }^{2}$ où se réunissent les Métis pour les offices religieux, puis il aménage dans une hutte misouterraine dans le bord de la côte juste au nord de l'endroit où se construira bientôt l'église. En 1883, une autre petite maison est bâtie sur le terrain de l'église, mais les couleuvres en chasseront le prêtre. Il aura, en 1887, une maison en rondins mais assez spacieuse et à deux étages, demeure qu'il passe plus tard à la famille James Laboucane afin qu'elle soit partiellement à son service, tandis que lui-même occupe un appentis ajouté à cette maison. En 1925 seulement, un presbytère bien à lui s'élève auprès de l'église.

Cette dernière, guère plus grande qu'une chapelle, est construite en 1883. Elle n'est rendue étanche contre la pluie et le vent que petit à petit. Un Blanc canadien-français du nom de Camille David y peint des scènes artistiques sur la voute et les murs, tandis que le curé en compose sur les murs au moyen de morceaux de prélart découpé, de vieux ornements et même de bouchons de bouteilles de liqueur douce ou de bière, ces derniers décorés de têtes de buffalos. Cette église est devenue un musée par suite des efforts de l'organisation locale de l'Alberta Historical Society.

L'endroit portait tout d'abord le nom de Notikiwin Seppe ou Rivière Bataille, du fait que la vallée était l'endroit où plusieurs rencontres armées entre les Cris et les Pieds-noirs avaient eu lieu. Les Blancs le connaissent sous le nom de Battle River Crossing, car deux gués pour le passage des charrettes y existent. Il devient Salois Settlement en 1875 avec l'arrivée d'Abraham Salois et de quelques familles métisses, puis Laboucane Settlement en 1878 quand six familles de ce nom s'y établissent ${ }^{3}$.

2 Le vrai nom est « Lafournaise ", mais les anglophones, ne pouvant le bien prononcer, le changent en "Laboucane".

3 En 1875, François Dumont et Abraham Salois, venant du Lac Sainte-Anne, s'y établissent. Trois ans plus tard, ou en 1878, un groupe d'autres Métis laisse la Prairie du Cheval Blanc ou Saint-François-Xavier, près de Saint-Boniface, et vient s'ajouter à la petite population. Il s'agit surtout de cinq frères Laboucane, de Maxime Poitras et d'un nommé Saint-Germain - ces deux derniers mourant en route durant un voyage de quatre-vingt-dix jours et plus en charrettes de la Rivière Rouge - et de leurs familles. Un sixième frère Laboucane, établi au Dakota du Nord, est persuadé par Edmond Brosseau, fondateur du village du même nom sur les bords de la Rivière Saskatchewan du Nord au nord-est d'Edmonton, de fuir les Etats-Unis où règnent alors les guerres d'annihilation des Indiens, pour venir se joindre aux autres. Leurs noms sont Jean, Gabriel, Guillaume, Jérôme, Pierre et 
La division du territoire en lots de rivière, si chère aux Métis, sera fait accompli en 1883. Cela attire plus de familles encore, au grand bonheur du missionnaire qui aime de beaucoup mieux cette population qui, au maximum atteindra le chiffre de deux cent cinquante, que les Indiens ${ }^{4}$. Même, il devient Métis avec eux ${ }^{5}$. Les morts parmi eux l'affectent grandement ${ }^{6}$, il est des plus patient avec eux, tandis qu'il est moins tendre pour les Blancs qui scandalisent parfois ses Métis ${ }^{7}$; il est patient surtout avec Jean Tobie Laboucane, fils de James. Quand ce jeune prend une partie des quêtes, lèche les burettes durant la messe, boit du vin et le remplace par de l'eau, et finalement vend sans permission l'automobile Chevrolet du missionnaire, le curé l'excuse toujours. En 1914, en partance pour son pays natal où il doit subir une opération, il dit avec un sourire "Je m'en vais chez moi ", mais il y est à peine qu'il s'ennuie et il veut revenir "chez moi " au plus tôt ${ }^{8}$. Lorsque, surtout depuis 1898, ses Métis, dépourvus du frettage, leur gagne-pain principal, par le prolongement des chemins de fer, et gênés par le nombre d'immigrants blancs, partent petit à petit de Duhamel, ses lettres, qui deviennent de plus en plus courtes, laissent deviner un certain cafard, un certain regret de l'âge d'or.

En 1887, lors d'un voyage de plusieurs personnalités de l'Est vers les missions de $\mathrm{M}^{\mathrm{gr}}$ Durieu, o.m.i., en Colombie-Britannique, $\mathrm{M}^{\mathrm{gr}}$ Grandin parle de la mission de monsieur Beillevaire à $M^{\mathrm{gr}}$ Thomas Duhamel d'Ottawa. Ce dernier consent que son nom s'attache à la mission de la Rivière Bataille et il promet une cloche pour l'église. C'est ainsi que la Colonie Laboucane est, dès lors, connue sous le nom de Saint-Thomas-deDuhamel, nom donné aussi au bureau de poste ouvert en 1892. La cloche arrive au printemps de 1893.

Bien qu'il y ait cinq magasins sur place, car trois des frères Laboucane en ont chacun un, un Américain en possède un géré par

Elzéar. Les trois premiers prennent du terrain au nord de la rivière, tandis que les trois autres le font au sud. Dès lors la colonie porte leur nom.

En mai 1881, Samson Breland, de Saint-François-Xavier aussi, et d'autres charroyeurs se dirigent vers l'Ouest, passent par ce qui est Moose Jaw et arrivent à la fourche des Rivières la Biche (Red Deer) et la branche sud de celle appelée Saskatchewan. Là se joignent à eux les familles de Pierre et de Gabriel Léveillé et de trois frères Poitras. A leur retour du Fort des Prairies ou Edmonton, l'abbé Beillevaire, déjà établi chez Elzéar Laboucane, les rencontre à la traverse de la Rivière Bataille, parlemente avec eux et les persuade de demeurer sur place.

4 A l'avenir, dans les apostilles, L signifie * lettre »; LL, lettres *;

$\mathrm{HB}$, Hyppolite Beillevaire; JMB, Jean-Marie Beillevaire, frère du missionnaire;

M, Mariette, sœur de HB; B, Bernadette, sa nièce, à Sainte-Pazanne.

L, HB à JMB, 25 avril 1884.

5 Témoignage de D. R. Francœur de Camrose donné à E. 0. Drouin, 1952.

$6 \mathrm{~L}, \mathrm{HB}$ à JMB, 19 janvier 1884; L, HB à JMB, 10 mars 1885; LL, HB à JMB, 10 novembre 1886,15 janvier 1888 , 4 juillet 1896 .

7 L, HB à JMB, 16 mars 1882 ; témoignage de $M$. et $M^{\text {me }}$ John Martz donné

à E. O. Drouin,

8 Témoignage de $M$. et $\mathrm{M}^{\mathrm{m} e}$ John Martz donné à E. O. Drouin en 1952. 
Léon Paré dont l'épouse est Marie Laboucane, et un autre est sous l'égide de la Compagnie de la Baie d'Hudson chez les Salois, le missionnaire s'approvisionne surtout au Fort Edmonton lors de ses voyages plutôt nombreux dans cette direction. A partir de 1890, lorsque François Adam, un ingénieur belge, établit un autre magasin dans le fond de la vallée, d'autres Blancs arrivent et un petit hameau contenant un peu de tout - magasins, hôtel, boutique de forge, bureau des postes, bureau de médecin, poste de la Gendarmerie à cheval, une laiterie, un caravansérail, etc., sort de terre. Aujourd'hui, rien de tout cela subsiste. Le terrain est un endroit de campement pour touristes.

Au moment où l'abbé arrive à Duhamel, il n'y a guère que trois centres missionnaires au sud de la branche nord de la Rivière Saskatchewan - Battleford, Bow River (Calgary) et la Rivière Bataille ${ }^{9}$. Les Indiens, les Métis et les Blancs catholiques doivent s'y rendre pour obtenir des secours religieux, ou bien il faut que les prêtres rayonnent à de grandes distances pour les rejoindre. C'est bien ce que Beillevaire doit faire constamment, surtout au temps des Pâques, et pour soulager les malades spirituellement mais même médicalement car, durant une longue période de temps, aucun médecin n'existe en dehors du Fort Edmonton ${ }^{10}$.

Les noms modernes des endroits où il va pour le ministère sont les suivants: Bashaw ou Lac la Biche; Camrose; Earling; Edberg; Gwynne ou Belle Colline; Hardisty; Hobbéma; les Lacs Bois Blanc, Butor, Castor, des Foins ou Grands Marais, des Pins, Pigeon. Rond, Salé, et Qui-ne-Gèlepas; la Butte et le Lac de la Viande sèche; Lewisville; New Norway; Pipe Stone Creek; Rivière la Queue et Lac du Bœuf ou Buffalo: Rivière Taille; Rosenroll; Spring Lake; Rosalind; Tofield; Vegreville: Wetaskiwin. Liste d'autant plus impressionnante que tous les voyages, excepté ceux des dernières années de sa vie. se font avec des chevaux et par tous les temps. Beaucoup d'entre eux sont pour répondre à des appels aux malades. Dans ces cas, il n'hésite jamais et il part aussitôt que la nouvelle lui arrive, souvent même au péril de sa vie ${ }^{11}$. Sa promptitude et son dévouement sauvent des vies et surtout des âmes ${ }^{12}$. Il est un des prêtres qui croient fermement que son ordination sacerdotale le rend apte à opérer des miracles de guérison et même de prophétiser l'avenir ${ }^{13}$. Pas satisfait de tout cela, il prêche aussi des "missions" aux Indiens avec le père Scollen et le père G. Simonin ${ }^{14}$.

9 Article dans le Western Catholic, journal d'Edmonton, 21 juillet 1921.

10 LL, HB à JMB, 10 novembre 1886, 3 avril 1889, 26 février 1890.

11 LL, HB à JMB, 27 juin 1888, 3 avril 1889, 12 mai 1891.

$12 \mathrm{~L}, \mathrm{HB}$ à JMB, 3 novembre 1905; LL, HB à M, 30 novembre 1892, 18 février 1907, 9 mars 1908; témoignage d'Álexander Ross donné à E. O. Drouin en 1952.

13 Témoignage de $\mathrm{M}$. et $\mathrm{M}^{\mathrm{me}}$ John Martz donné à E. O. Drouin en 1952.

$14 \mathrm{~L}, \mathrm{HB}$ à JMB, 27 novembre $1895 ; \mathrm{L}, \mathrm{HB}$ à $\mathrm{M}, 10$ août 1922. 
Ces courses éreintantes ne se terminent qu'à sa quatre-vingt-quatrième année, de sorte qu'il ne mène certes pas une existence de tout repos.

La vie quotidienne demande beaucoup de renoncement. On se tire d'affaire comme on le peut avec de maigres ressources. Ainsi, durant un certain temps au cours du premier hiver de Beillevaire dans sa hutte mi-souterraine, le froid intense, une neige très épaisse et des tempêtes répétées l'empêchent même de sortir. Il doit recourir au stratagème de placer une boîte avec une porte de deux côtés sur le seuil de la fenêtre. Lorsqu'un petit oiseau de neige, attiré par quelques graines, y pénètre, le prêtre, au moyen d'une corde, fait retomber le côté extérieur de la boîte, ouvre la fenêtre et tord le cou du petit être afin de s'en faire de la soupe ${ }^{15}$. Le commandement de l'abstinence n'est pas urgé mais on doit l'observer forcément plus souvent que le vendredi, bien des fois.

Il n'est certes pas au poste dans le but de s'enrichir. De 1881 à 1889 , il peut compter sur un octroi annuel de $\$ 300$ de la part de $\mathbf{M}^{\text {gr }}$ Grandin, mais depuis lors il doit se débrouiller seul. Ses Métis ne peuvent guère l'aider, car leur pauvreté est grande. Il leur demande le moins possible, et même, au contraire, c'est lui qui les aide souvent ${ }^{16}$. Il doit trouver linge, remèdes et honoraires de messes en France. Tant que son frère l'abbé Jean-Marie vit, c'est lui qui se fait le pourvoyeur principal, mais quand une mort prématurée l'enlève, ce seront sa sœur Mariette et Bernadette, fille de cette dernière, qui prendront la relève ${ }^{17}$. Entre autres, son rapport financier de 1918 indique un déficit de $\$ 51.70$ pour janvier et février seulement; celui de 1922 montre qu'il a un surplus de $\$ 2.80$ au 31 décembre. Évidemment, personne n'a jamais dévalisé sa maison! A sa mort en 1937, après cinquante-sept ans de travail, il n'a que $\$ 314$ ì son nom ${ }^{18}$.

Si le gain matériel n'est pas le mobile de son apostolat, comme la chose est plus qu'évidente, quel serait-il ?

Bien sûr, ce n'est pas le snobisme qui l'anime. Issu d'une famille des plus importantes de Sainte-Pazanne où l'on découvre des maires, des médecins, des pharmaciens, des ingénieurs, etc., il ne prétend jamais étre sur le haut d'un piédestal d'où il commande orgueilleusement la

15 L, HB à JMB, 28 janvier 1882; témoignage de $M^{\text {me }}$ Joseph Martz donné à E. O. Drouin en 1952.

16 Témoignage de $\mathrm{M}^{\mathrm{me}}$ Hutchinson donné à E. O. Drouin en 1952.

17 LL, HB à JMB, 19-11-1883, 26-8-1884, 16-11-1886; LL, B à HB, 22-12-1915, 1-3-1917, 22-3-1918, 13-6-1918, 26-12-1918, 10-4-1919, 20-9-1919, 9-2-1925, 10-2-1927, 8-3-1929, 29-12-1934, 2-5-1933, 12-5-1933, 16-8-1933, 17-9-1933, 18-1-1934, 5-3-1934, 30-4-1934, 3-9-1934, 16-11-1934, 12-12-1934, 18-1-1935, 29-1-1935, 24-2-1935, 25-3-1935, 24-4-1935, 25-7-1935, 5-10-1935; L. A. Naessens, o.m.i. à HB, 3-8-1929; LL, HB à B, 24-3-1934, 23-1-1936, 7-1-1937; mot de HB à son $86^{\circ}$ anniversaire, 21-1-1934.

18 L, Pierre Moulin, o.m.i., à B, 10-3-1937. 
déférence et le respect. Les gens l'ont sans cesse en très grande estime mais seulement parce qu'il la gagne, cette estime, car il est humain, sociable, joyeux, charitable, sensible, dévoué et humble. Il est aussi pieux et totalement donné à Dieu et à son devoir d'état.

C'est avec raison qu'un ami, en mesure de l'observer durant des décades, affirme, quelque quinze ans après sa mort: "Il était un saint, tout comme sa vie le prouvait. Il n'était toutefois pas scrupuleux, mais il avait une foi vive... Il n'était pas vantard mais bien plutôt fort humble. Tous l'aimaient et tous allaient à lui avec une confiance absolue, même les protestants. Il s'était fait Métis avec les Métis ${ }^{19}$." On pourrait avoir plus mauvaise réputation!

Déjà, au séminaire de Nantes ${ }^{20}$, puis lors de son départ de France pour les missions ${ }^{21}$, et encore lorsque sa mère et sa sœur sont rappelées à la Maison du Père ${ }^{22}$, il révèle l'esprit surnaturel qui est le mobile de sa vie. Il ne craint pas le sacrifice; il est imbu de l'amour divin. Ce sont là des vertus essentielles pour tout apostolat solide.

Sa charité est proverbiale. Il ne peut jamais refuser à qui tend la main ${ }^{23}$. Pour souligner son humilité ne citons que deux extraits, les deux de lui-même. Le premier date de ses noces d'or sacerdotales. Il y est sincère et véridique ${ }^{24}$ :

Il y aura donc 41 ans que je suis ici. Pendant ce temps j'ai fait quelque bien, oh ! s'il en était autrement, ce serait pire que le serviteur inutile, mais je m'empresse de le dire, avec plus d'énergie, plus de piété et de zèle, j'en aurais fait bien davantage. Ceci est la vérité, je le vois bien maintenant. Pour m'encourager j'ai l'Evangile des ouvriers de la dernière heure, aussi avec la grâce de Dieu, la protection et l'assistance de la $S^{\text {te }}$ Vierge, j'espère atteindre la couronne dont parle $S^{t}$ Paul.

\section{Témoignage de D. R. Francœur donné à E. O. Drouin en 1952.}

20 HB, Règlement particulier.

21 L, HB à JMB, 30-1-1885; L, HB à M, 30-1-1885.

22 L, HB à JMB, 30-1-1885; L, HB à B, 17-11-1927.

23 Il donne un de ses deux chevaux à Charles Descheneau dont la tente et tout l'avoir est la proie des flammes (témoignage de $\mathbf{M}^{\mathbf{m e}}$ Béatrice Busness donné à E. O. Drouin en 1952); il prélève de ses maigres vivres pour soulager la faim de familles diverses; il sadopte, à distance une orpheline coréenne du nom de Rosa (L, S $\mathbf{r}$ M. Donatienne, Taïkou, Corée, 6-1-1919); il envoie toujours quelque chose aux quémandeurs dont les demandes lui arrivent d'un peu partout (Document \# 214 . Beillevaire * aux Archives 0.M.I.'s, Edmonton, résumant nombre de telles demandes); il craint que sa sœur Mariette et sa nièce Bernadette, surtout durant la première Grande Guerre et les deux dépressions qui la suivent, soient trop privées du nécessaire à leur existence, alors et bien des fois il les enjoint de prélever sur ce qu'elles quêtent pour lui afin de suffire à leurs besoins (LL, B à HB, 30-10-1918; 6-11-1918; 29-11-1918; 26-12-1918); il prête même ses chaussures et chaussettes au médecin de Duhamel; enfin, lorsque la vieille $M^{\text {me }}$ Martz se charge de recueillir les fonds voulus pour acheter une statue du Sacré-Cour qu'il désire grandement, elle refuse de lui donner l'argent - elle veut payer la facture elle-même - car elle sait d'expérience que cette statue ne se rendra jamais à Duhamel. Le curé se serait servi du montant pour soulager des misères (témoignage de $\mathbf{M}$. et $\mathbf{M}^{\mathbf{m}}$ John Martz donné à E. O. Drouin en 1952).

24 Adresse lue par HB à $M^{\text {gr }}$ Joseph Henry 0'Leary, 30-8-1922. 
Dans le deuxième cas, il s'avoue coupable devant un jeune séminariste du nom d'Emmett Engel qui, en 1928, y est pour enseigner du catéchisme de vacance, et qui vient justement de terminer le nettoyage du sanctuaire: - Depuis si longtemps que je manque de respect et d'amour envers Notre-Seigneur ! Je vais prier et lui demander pardon ${ }^{25}$."

Son humilité ne supprime toutefois pas le fait qu'en bon Breton il soit têtu ${ }^{26}$. Ce défaut devient vertu lorsqu'il s'agit pour lui de prendre des risques par tous les temps et malgré les conseils de ses gens afin de voler au secours des malades, peu importe les distances. Il faut dire, cependant, qu'en hiver il voyage rarement seul, même s'il est doué d'un sens spécial des directions ${ }^{27}$.

Son tempérament breton, ou faudrait-il dire sa dévotion à la mère de la Vierge, paraît encore lors du pèlerinage annuel à Sainte-Anne au lac du même nom, même s'il lui faut parcourir deux cent quatre-vingts milles pour y prendre part ${ }^{28}$. Il en est de même pour sa participation de tous les ans à la retraite du clergé. Cela lui est sacré ${ }^{29}$, et l'âge ne le retient jamais.

Il n'est pas contemporain de notre monde postconciliaire où on boute dehors à peu près toutes les dévotions, soi-disant parce qu'il faut concentrer sur la messe et les sacrements. Pour lui-même et pour ses paroissiens - et cela touche un point de pastorale de chrétienté - il veut une atmosphère facilitant la compréhension de l'amour divin dans la messe et les sacrements. Dans ce but, il encourage tout le monde à se rendre aux dévotions suivantes: celles des Vêpres et de la Bénédiction du Très Saint Sacrement les dimanches; celles du Sacré-Cour et du premier vendredi du mois; celle de Marie - un témoin affirme que jamais il n'a entendu parler de la Mère de Dieu avec autant de ferveur, d'amour et de persuasion - et celle du chapelet. En plus, presque tous

25 Témoignage de $M$. et $\mathbf{M}^{\text {me }}$ John Martz (Eléonore Engel, sœur d'Emmett), donné à E. O. Drouin en 1952.

26 Emilie Laboucane, sa ménagère et femme de James, en sait quelque chose, surtout quand elle veut redresser son logis et faire disparaître les douzaines et les douzaines de boîtes vides de snuff - en 1952, il en existe encore toute une famille dans l'appentis de la maison Laboucane - ou encore lorsque, durant les dernières décades de la vie de Beillevaire, elle insiste parfois à lui laver les mains, la figure et les cheveux, quand il eut renoncé presque totalement à descendre à la rivière pour y nager comme un poisson. Elle a tempêtait souvent contre lui », affirment des témoins, mais alors il lui disait: - Pouah ! Quand je t'ai vue la première fois à la traverse de la rivière, en 1881, il n'y avait pas moyen d'avoir un mot de toi; tu te cachais derrière ta sœur. Maintenant ta langue n'arrête pas et elle ne parle plus !,

27 Témoignage de $M$. et $\mathbf{M}^{\mathrm{me}}$ John Martz donné à E. O. Drouin en 1952.

28 L, HB à JMB, 27-7-1891; L, Emile Legal à JMB, 15-9-1899; L, HB à B, 31-7-1928; Journal quotidien, $M^{\mathrm{gr}}$ Emile Legal, 28-6-1924; Rapport de Z. Lizée, o.m.i., au Supérieur général, 23-7-1895, dans Les Missions de la Congrégation des Missionnaires Oblats de Marie-Immaculée, Paris, Typographie A. Hennuyer, livraison de décembre 1896, p. 490-491.

$29 \mathrm{~L}, \mathrm{HB}$ à une demoiselle amie de la famille en France, 19-10-1884; LL, HB à JMB, 4-7-1896; 11-10-1901; 1905; L, B à HB, 24-6-1933. 
font partie de la confrérie de Saint-Joseph pour la bonne mort ${ }^{30}$, et de celle de Sainte-Barbe ${ }^{31}$. En plus encore, la Ligue des Femmes catholiques prend naissance en 1922 et continue ses activités après la mort de Beillevaire.

Bien que ne commandant pas l'autorité pour sa personne d'une façon cassante, il est fort déférent pour ceux à la gouverne civile et ecclésiastique. Cette attitude chez lui, sa façon d'agir avec et devant ses ouailles ou toute la population, ses interventions et ses paroles cultivent chez ses gens et même dans tout le territoire sous sa juridiction la même psychologie, de sorte que, même aujourd'hui, on lui attribue l'esprit de concorde, de paix et d'unité régnant à et autour de Duhamel ${ }^{32}$.

Ainsi, au cours des années 1881 à 1884, lorsqu'il est responsable des Indiens d'Hobbéma, il ne manque jamais d'être présent aux réunions des tribus quand a lieu le "traité " ou la distribution des argents promis par le gouvernement canadien aux autochtones. Il y est pour aider à maintenir l'ordre et la paix, pour voir aussi parfois à ce que ses enfants des prairies ne soient pas poussés à bout par certains agents sans scrupule. Cela a alors une fort grande importance, car c'est une période de famine ou presque ${ }^{33}$. En juillet 1883, il se fait un devoir de circuler dans le camp indien qui, près du Fort Edmonton, manifeste d'une façon plus menaçante que d'habitude son déplaisir envers les hommes du gouvernement. Il faut absolument persuader les Indiens de ne pas commettre d'excès, mais il faut encore défendre leurs droits ${ }^{34}$.

De même, en collaboration avec d'autres prêtres, surtout avec le père Scollen, il sillonne la plaine durant l'année 1885 et il visite les familles individuelles et organise des assemblées publiques afin de maintenir la paix et d'empêcher Indiens et Métis de joindre leur force à celle de Louis Riel. Cependant, lorsque certaines têtes chaudes de parmi les siens, aiguillonnées par une poignée d'émissaires de la Saskatchewan, venus se réfugier dans les parages à la suite de la première escarmouche entre Métis et la Gendarmerie à cheval près du Lac Canard de la province voisine, pillent le magasin géré par Léon Paré en 1885, il n'hésite pas un moment à monter à cheval. Il traverse la vallée. Sans crainte il s'introduit au sein du groupe fêtant la "victoire ". Longtemps il parlemente et il les persuade finalement de rendre le plus de butin possible ${ }^{35}$. Bien plus tard la même année, une situation beaucoup plus

$30 \quad$ L, HB à B, 8-3-1929.

31 LL, HB à B, 31-7-1928, 15-8-1932; LL, B à HB, 30-3-1933, 5-10-1935.

32 Témoignage de $\mathrm{M}^{\mathrm{me}}$ Hutchinson donné à E. O. Drouin en 1952; témoignage de $\mathbf{M}^{\mathrm{me}}$ Everet Chevraux donné à E. O. Drouin en 1952.

33 LL, HB à JMB, 27-8-1881, 7-7-1882, 10-12-1882, 12-10-1883; L, HB à une demoiselle de France, amie de la famille, 19-10-1884.

$34 \mathrm{~L}, \mathrm{HB}$ à JMB, 27-7-1883.

$35 \mathrm{~L}, \mathrm{HB}$ à JMB, 15-6-1885; témoignage de $\mathrm{M}^{\mathrm{me}}$ Béatrice Busness donné à E. O. Drouin en 1952 . 
tragique se présente et, cette fois, il sauve la vie à quarante-deux membres de la Gendarmerie ${ }^{36}$.

Si l'abbé Beillevaire enseigne ainsi à ses gens le respect de l'autorité civile, il fait encore plus vis-à-vis de celle de l'Église. Par exemple, à chaque visite de $\mathrm{M}^{\mathrm{gr}}$ Grandin ${ }^{37}$, de $\mathrm{M}^{\mathrm{gr}}$ Émile Legal ${ }^{38}$, il organise toute une cavalcade qui rencontre les évêques à la Belle Colline, le Gwynne actuel, pour les escorter en pompe et au bruit d'une mousqueterie bien fournie jusqu'à l'église à une douzaine de milles de là. Tout est fait aussi grandiosement que possible durant les cérémonies de la confirmation. D'ailleurs, cela plaît fort aux Métis qui aiment les spectacles, les processions et les couleurs voyantes. Depuis le temps où $\mathbf{M}^{\text {gr }}$ O'Leary succède à $\mathbf{M}^{\mathrm{gr}}$ Legal en 1920 , il s'en tient toutefois pratiquement aux célébratioñis à l'église, car on voyage en automobile ex les cavaícades doivent être plutôt abrégées ${ }^{39}$.

36 Nonobstant une promesse solennelle, Noël Salois, en secret, organise un groupe de Métis qui part à la dérobée en direction de Batoche, Saskatchewan, afin d'y épauler Riel et les siens. Leur enthousiasme ne semble toutefois pas tellement fort car ils voyagent à très petites journées et ils n'arrivent qu'après la fin des hostilités. Malgré cette tournure de choses, la Gendarmerie envoie quarantedeux hommes à cheval pour faire perquisition dans les maisons à Duhamel afin d'y confisquer les armes et arrêter des hommes compromis. Evidemment, leur venue est signalée d'avance. Ils ne découvrent ni fusils ni hommes. Presque toutes les familles se sont réfugiées ici et là dans les vallées et les ravins multiples. Pour son compte, Noël a pris la poudre d'escampette juste à temps. A dos de cheval, il s'est esquivé vers les Etats-Unis. Bredouilles, les policiers rebroussent chemin vers le Fort Edmonton. Sans tentes, le premier soir, ils font bivouac à quelques milles sur les bords du Lac Butor ignorant le danger qui les menace. En effet, à distance, ils ont été espionnés par une quarantaine de jeunes gens qui se préparent à faire un mauvais parti aux redingotes rouges. Leur plan est d'attendre la tombée de la nuit pour s'introduire à pas feutrés dans le campement, d'enlever tous les chevaux, de se cacher ensuite à quelque distance, de créer un tintamarre afin d'induire les gendarmes à sortir de sous le bois. Ainsi, ils pourront être abattus sans pouvoir se défendre. Averti par des femmes inquiètes, Beillevaire saute sur un cheval et, au galop, se dirige vers l'endroit où il devine que ses Métis se cachent. Il les découvre. Durant une heure environ, il leur parle de la folie de l'entreprise. * Ces policiers, leur dit-il, ne sont personnellement coupables de rien; ils ne font qu'obéir aux ordres d'enlever les armes et d'arrêter Gabriel Dumont et Alexandre Maukomosik (Couteau de Poche), deux hommes de Riel, et Noël Salois qui vous a entraînés malgré ses promesses. Ils n'en veulent ni aux Indiens ni aux Métis. Leur seul but est de maintenir l'ordre et la paix dans le pays. Vous pouvez très facilement les tuer tous, mais soyez sûrs que le gouvernement enverra d'autres soldats pour vous faire tous prisonniers et vous pendre. Qu'adviendra-t-il alors de vos femmes et de vos enfants? En maugréant, les aventuriers suivent leur missionnaire. Il les livre un à un à leurs femmes auxquelles il donne ordre de voir à ce que les maris ne quittent pas les maisons jusqu'à ce qu'il revienne lui-même leur enjoindre de a libérer > leurs hommes. (Cf. L, HB à JMB, 15-6-1885; $\mathbf{M}^{\mathrm{m}}$ e Hutchinson, extrait de ses documents personnels; témoignage de $\mathbf{M}^{\mathrm{me}}$ Joseph Martz donné à E. O. Drouin en 1952.)

37 LL, HB à JMB, 23-1-1889, 3-4-1889; adresse lue par HB à $\mathrm{M}^{\mathrm{gr}} \mathrm{J} . \mathrm{H}$. O'Leary, 30-8-1922.

38 L, HB à JMB, 7-6-1893; L, Emile Legal à JMB, 15-9-1899; témoignage d'Alexander Ross donné à E. O. Drouin en 1952.

39 Adresse de HB à $\mathrm{M}^{\mathrm{gr}} \mathrm{J}$. H. O'Leary, 30-8-1922; L, HB à M, 6-9-1922. 
Le contenu d'un certain nombre de paragraphes précédents et ce qui suit immédiatement soulignent un point de pastorale d'évangélisation employé généralement par à peu près tous les missionnaires du temps. En effet, ils ne peuvent alors se contenter de rester dans leur presbytère, ou mieux leur cambuse, attendant que les paroissiens viennent à eux. Leurs relations et leurs activités humaines et sociales sont de tous les jours. En d'autres mots, ils donnent en plein dans ce qu'on appelle maintenant « le projet humain " mais, même s'ils ignorent la signification du mot " humanisme " tel qu'il a cours aujourd'hui, ils évitent de donner dans cette erreur. Le but de leurs efforts est de s'intéresser à tout ce qui est vital pour leurs gens afin de l'employer pour conduire ces derniers au niveau surnaturel. Leur réussite n'est pas toujours mirobolante, bien entendu, car la nature humaine est peut-être plus nature humaine chez les Indiens et les Métis que chez d'autres.

Beillevaire non seulement permet à son monde de se sentir tout à fait chez lui dans sa maison ${ }^{40}$, mais lui-même visite constamment toutes les familles, mange et couche chez elles, sur le plancher au besoin; participe à toutes les noces ${ }^{41}$; reçoit toutes les familles le Jour de l'An, et un bon nombre aux Rois mangent avec lui; invite un couple tous les dimanches à venir dîner avec lui, mais cela est surtout un truc ${ }^{42}$ pour qu'une femme lui prépare un bon repas par semaine et lave toute la vaisselle salie durant les sept jours précédents. Sa passion pour le jeu de cartes, surtout pour le 500, souvent l'introduit dans bien des familles, en particulier mais pas exclusivement chez les protestantes ${ }^{43}$. Est-ce étonnant si, durant tout son séjour de cinquante-six ans à Duhamel, il n'y a point de naissances illégitimes et aucun crime sérieux ?

Dans un autre domaine touchant la pastorale d'évangélisation, il sst de beaucoup avant son temps. Il s'agit des rapports écuméniques (interfaith) avec les quelques familles protestantes qui arrivent peu à peu. Les documents ne mentionnent que deux conversions au catholicisme parmi elles ${ }^{44}$, mais le degré de bonne entente qu'il engendre n'est point à dédaigner.

Il les visite tout autant qu'il le fait pour ses catholiques; il leur rend des services ${ }^{45}$; il les invite tous non seulement aux banquets de ses divers

40 L, HB à une demoiselle de France, amie de la famille, 26-2-1888; LL, HB à $\mathrm{B}$, 20-1-1929, 27-2-1937.

41 LL, HB à JMB, 3-2-1886, 27-11-1895.

42 LL, HB à JMB, 3-1-1883, 18-1-1884; L, HB à demoiselle, 26-2-1888; L, HB à B, 20-1-1929.

43 L, HB à demoiselle, 26-2-1888.

44 Témoignage de $M^{\text {me }}$ Fred Dittberner, 1952; témoignage de $M^{\text {me }}$ Joseph Martz et témoignage de D. R. Francœurs donnés à E. O. Drouin, 1952; témoignage de Sour Marie-Adèle de l'hôpital de Camrose, 9-1-1962.

45 Témoignage de $\mathbf{M}^{\text {me }}$ Freda Smith donné à E. O. Drouin en 1952. 
anniversaires et de ses noces sacerdotales ${ }^{46}$, mais même à sa procession annuelle. Sa charité et sa bonhomie gagnent leurs cours. Ces noncatholiques vont nombreux à ses fêtes, fournissent de la nourriture et servent les tables avec les autres ${ }^{47}$; des notables de Camrose s'y rendent de même ${ }^{48}$; ils assistent tous à la procession de la Fête-Dieu ${ }^{49}$, jour où leur propre service qui a lieu au premier étage d'un magasin du hameau est supprimé en faveur de la cérémonie sur le haut de la côte le soir ${ }^{50}$. Chose plus étonnante peut-être est le fait qu'ils fournissent leur quote-part pour acheter la statue du Sacré-Cœur mentionnée plus haut ${ }^{\mathbf{5 1}}$. La famille Alexander Ross, occupant le domaine jadis propriété de Jérôme Laboucane, a une confiance absolue en lui. Comme tout le monde, elle considère Beillevaire comme le meilleur paratonnerre ou plutôt la parfaite garantie contre la grêle ${ }^{52}$. On attribue cela à ses prières et à la tournée pour bénir toutes les fermes aux Rogations. A preuve, une journée où ce fléau menace, une des filles aperçoit le prêtre se promenant sur la route. Elle annonce aux siens qu'il n'y a rien à craindre, car le missionnaire récite ses prières dans son livre. D'ailleurs, cette réputation existe depuis bien longtemps puisque, affirme-t-on, jamais la grêle ne tombe ou au moins ne cause de dommage à Duhamel tout le temps que Beillevaire y demeure. Encore aujourd'hui, Alexander Ross se rappelle avec émotion l'esprit ouvert et la grande charité du prêtre prouvés, entre autres, par le fait qu'au temps de la maladie ultime de son père, Beillevaire vient très souvent l'encourager et prier avec lui. De plus, la mort étant survenue, il monte son autel portatif dans la maison protestante et y offre le Saint Sacrifice pour le repos de l'âme du défunt ${ }^{53}$. Lorsque le prêtre, avant de l'avoir chez lui, veut faire usage du téléphone, c'est chez Ross qu'il se rend. Ce dernier raconte comment, la première fois, Beillevaire fut tout estomaqué lorsqu'il entendit $\mathrm{M}^{\mathrm{me}}$ Adam de Camrose lui parler en français. Il croyait que tout probablement un téléphone dans une maison protestante et anglaise ne pouvait "parler" qu'anglais ${ }^{54}$.

Est-ce étonnant si un protestant qui l'a bien connu affirme " qu'il était le meilleur des amis ${ }^{55}$ ", et tous les témoins sont d'accord pour chanter ses louanges, surtout pour dire qu'il était responsable de la paix et de l'esprit d'entraide dans tout le territoire ${ }^{56}$.

46 Témoignage de $M$. et $M^{\text {me }}$ John Martz donné à E. O. Drouin en 1952.

$47 \mathrm{LL}, \mathrm{HB}$ à M, 7-1.1916; adresse, HB à $\mathrm{M}^{\mathrm{gr}} \mathrm{J}$. H. O'Leary, 30-8-1922;

LL, HB à B, 25-1-1932, 15-8-1932, 10-2-1933.

48 Adresse, HB à M ${ }^{\mathrm{gr}}$ O'Leary, 30-8-1922; L, HB à M, 20-10-1922.

49 Témoignage d'Alexander Ross donné à E. O. Drouin en 1952.

50 Témoignage d'Alexander Ross donné à E. O. Drouin en 1952.

51 Témoignage de M. et $M^{\text {me }}$ John Martz donné à E. O. Drouin en 1952.

52 Témoignage de $M^{\text {me }}$ Hutchinson donné à $E$. 0 . Drouin en 1952.

53 Témoignage d'Alexander Ross donné à E. O. Drouin en 1952.

54 Témoignage d'Alexander Ross donné à E. O. Drouin en 1952.

55 Témoignage de $M^{\mathrm{me}}$ Freda Smith donné à E. O. Drouin en 1952.

56 Témoignage de $\mathrm{M}^{\mathrm{m} e}$ Hutchinson donné à E. O. Drouin en 1952. 
Venons-en au domaine qui touche de plus près aux méthodes de la pastorale de chrétienté à Duhamel.

D'abord l'école. Entre 1881 et 1887, il n'en existe pas du tout. De 1887 à 1901, on en a une à proximité de l'église mais les professeurs et les résultats sont à l'avenant. Beillevaire voudrait pouvoir compter sur ces hommes pour donner aux enfants une instruction religieuse suivie et solide mais, même si lui-même y fait ce qu'il peut quand ses courses apostoliques ne l'éloignent pas, un doute assez sérieux demeure. A partir de 1901, l'école ayant été transportée et étant devenue publique ou neutre ${ }^{57}$, il doit de nouveau avoir recours à divers expédients pour les catéchismes. Il recommence ses anciennes habitudes de les faire soit dans les familles où il rassemble quelques enfants, soit au presbytère où il en convoque au moyen d'un cornet qu'il joue à l'extérieur vers les quatre points cardinaux ${ }^{58}$. Il ne peut plus compter sur les services d'Abraham Salois qui, dès l'arrivée du missionnaire à Duhamel, rendait de fiers services comme catéchiste laïc. Sur le tard dans sa vie, il adopte plutôt la méthode de faire donner l'instruction religieuse par une ou deux femmes avant la messe du dimanche, méthode toujours en vogue.

Quelques enfants métis vont toutefois au pensionnat indien d'Hobbéma depuis $1898^{59}$, et d'autres à la colonie Saint-Paul-des-Métis un peu plus tard ${ }^{60}$.

Serait-ce le résultat d'une pastorale pas assez en profondeur à cause des circonstances, ou de mauvaise volonté de la part de parents pas trop instruits de leur religion, ou encore du désir chez les humains

57 En 1887, Léon Paré, dans son magasin, situé à l'extrémité ouest de la colonie, fait la classe durant deux mois. C'est trop loin pour la plupart des enfants et alors, après bien des discussions, on se sert de l'église durant plus d'un mois tandis qu'on termine la construction d'un édifice en bois rond à proximité de celle-ci. Dès lors et durant trois années, Ambroise Grey, détenteur d'un certificat et originaire de Saint-Albert, est en charge (LL, HB à JMB, 3-9-1890, 31-12-1890; témoignage de $\mathrm{M}^{\mathrm{me}}$ Emilie Laboucane écrit en 1933). Lui succèdent Patrice Léveillé puis un nommé Girard. Avec lui, les enfants n'apprennent rien, car le maître les laisse faire à leur guise tandis que, les pieds campés sur le dessus du bureau plus souvent que de juste, il passe son temps à lire ou à ne rien faire en particulier. On change plutôt souvent de professeurs. Ainsi, on aura Arsène Bourque, Daniel Laboucane, Albert Laboucane et Dolphus Campion jusqu'à l'arrivée d'un Thériault qui préside à l'éducation des jeunes de 1898 à 1901. Plusieurs familles blanches, ayant pris des terrains au bout ouest de la colonie, ouvrent de nouveau la controverse au sujet de l'emplacement de l'école. Elles gagnent leur point et la maison est transportée sur la ferme actuelle de monsieur Ruttle. Dès lors, c'est une école publique où la religion n'a plus droit de cité. $M^{\text {me }}$ Hutchinson en est la première maîtresse durant l'année 1901-1902 (témoignage de $\mathbf{M}^{\mathrm{me}}$ Hutchinson donné à E. O. Drouin en 1952).

58 LL, HB à JMB, 28-1-1882, 3-2-1886, 15-1-1888, 28-1-1882 reproduite dans Les Missions de la Congrégation des Missionnaires Oblats de Marie-Immaculée, Bar-le-Duc, Saint-Paul, Paris, Typographie A. Hennuyer, rue d'Arcet, 7, dans la livraison de septembre 1882; L, HB à M, 14-6-1907.

$59 M^{\mathrm{gr}}$ E. Legal, Journal quotidien, 26-3-1898.

60 Témoignage de $\mathbf{M}^{\mathrm{me}}$ Béatrice Busness donné à E. O. Drouin en 1952. 
d'avoir quelque chose pour rien, mais, en tout cas, le problème scolaire et donc l'avenir religieux de plusieurs enfants se compliquent à partir de 1898. De fait, certaines familles se laissent persuader par un ministre anglican métis, nommé Matheson, de lui envoyer leurs jeunes à son école sur la réserve indienne du Lac d'Oignon. Il les prend gratuitement et les habille au besoin. C'est dire que l'abbé, malgré ses efforts et toute sa bonne volonté, ne réussit pas à faire de vrais catholiques de toutes ses ouailles. Rien de surprenant alors si le père J.-A. Thérien, o.m.i., fondateur et gérant de la colonie de Saint-Paul-des-Métis, n'aime pas trop voir lui arriver des gens de Duhamel ${ }^{61}$.

Quant à l'administration des sacrements et à la liturgie en général, monsieur Beillevaire a beaucoup plus de consolations. Toutefois, les multiples voyages requis dans des conditions parfois très difficiles les lui font gagner.

Puisqu'il offre souvent le Saint Sacrifice sous tente ou dans des maisons privées dans sa paroisse et un peu partout sur la prairie, les confessions sont plutôt sans cérémonie, mais elles sont nombreuses proportionnellement à la population, tout comme les communions d'ailleurs ${ }^{62}$. Même dans son église, il n'existe point de confessionnal durant bien longtemps. Ce n'est que peu de temps avant sa mort qu'il met un prie-dieu simple mais muni d'une grille. Habituellement, il s'asseoit dans une chaise berçante et les gens s'agenouillent à son côté. Une “jasette» a lieu, mais c'est surtout lui qui parle car, selon lui, la

61 Dans une lettre à Albert Lacombe, o.m.i., le père Thérien, en date du 8 février 1899, écrit: a Les Métis de la Rivière Bataille se préparent à laisser au printemps. Ils ne savent pas trop s'ils vont se placer dans la colonie ou à côté. Je crains ces gens, ils me semblent avoir des idées pas mal élastiques au sujet de leurs devoirs de chrétiens. Ce sont eux qui ont commencé le mouvement de mettre leurs enfants chez Matheson. Et je crains bien que ces nouveaux venus fassent de même. J'ai la douleur de vous apprendre qu'Elzéar Laboucane a mis son petit garçon chez Matheson malgré que les Sours lui eussent offert de le prendre pour rien. Vraiment ce sont de ces actes qui crient vengeance au ciel ... Il faut beaucoup de patience et de mansuétude tant que nous n'aurons pas une bonne école. Nous avons besoin d'être prudents si nous ne voulons pas voir passer au protestantisme quelques-uns de nos Métis. L'impression est répandue parmi eux que la plupart des prêtres ne s'occupent pas d'eux , (citée par E. O. Drouin dans Joyau dans la plaine, Québec, Les Editions Ferland, 1968, p. 151).

$\mathrm{M}^{\mathrm{gr}}$ Grandin se plaint des agissements de Matheson dans la paroisse de Duhamel: - Les Métis de cette localité nous ont causé récemment une peine sensible, et à laquelle nous ne pouvions nous attendre. Un ministre protestant leur ayant proposé d'élever leurs enfants gratuitement, ils se montrent très portés à accepter son offre. Quelques-uns même ont déjà eu la lâcheté de lui confier leurs enfants» ( $\mathrm{M}^{\mathrm{gr}}$ V. Grandin, Rapport paru dans Les Missions..., \# 141, livraison de mars 1898).

$M^{g r}$ E. Legal, à son tour, dépeint ce Matheson et ses activités d'une manière peu flatteuse. Il le dit peu scrupuleux dans ses méthodes pour attirer les enfants, même catholiques, à son école $\left(\mathrm{M}^{\mathrm{gr}} \mathrm{E}\right.$. Legal, Journal quotidien, 21-1-1898, 27-1-1898, 24-2-1898, 15-9-1905).

$62 \mathrm{HB}$, rapports à l'archevêché sur l'état du ministère de 1900 à 1928. 
confession consiste en une série de questions qu'il pose. Chacun répond " oui " ou "non " d'après le cas. C'est tout ${ }^{63}$.

La messe paroissiale du dimanche est quelque chose d'unique en son genre. D'abord, il insiste que ce soit un office chanté ${ }^{64}$, mais souvent, surtout aux grandes fêtes, avec accompagnement de violons grincheux, de musiques à bouche, de mandolines et de tappement de pieds par les " artistes ». Très tôt, après son arrivée sur place, il obtient un harmonium qu'il place près du petit sanctuaire. N'ayant pas d'organiste durant bien longtemps, il laisse l'autel à plusieurs reprises afin d'accompagner le chant. De sa belle voix de ténor, il chante lui-même des bouts de la messe. Afin d'épargner, il se fait des cierges quelconques, et, surtout, il n'en allume guère plus d'un à la fois.

A Noël, au Jour de l'An, aux Rois et à d'autres grandes fêtes, c'est fort solennel, et surtout l'église est surchargée de décorations et de bannières faites sur place avec du matériel de fortune ${ }^{65}$.

Combien longue est la messe? L'horaire indique qu'elle doit commencer à dix heures, mais cela n'arrive jamais. Les confessions terminées, le prêtre sort, se gare les yeux d'une main contre le soleil, même quand il n'y en a pas de visible, et il regarde dans toutes les directions pour voir si quelque voiture s'en vient. Il fait ainsi durant une période de temps plutôt prolongée sans que cela semble incommoder les Métis pour la peine car, pour eux comme pour les Indiens, le temps n'a point d'importance. Plus tard, les Blancs qui viennent s'ajouter à la population originelle s'impatientent de ce délai. Satisfait que tous sont enfin rendus, il va revêtir les ornements. Quelle heure est-il alors ? Onze heures peut-être, midi même. Du moment que la messe débute, jusquà l'Ite, missa est, il s'écoule entre une heure et demie et deux heures. Il prêche longtemps. Il le fait en cris, en français et en anglais. Tous ses sermons sont écrits et lorsque le temps arrive pour l'anglais - quel anglais horrible et barbare il $\mathbf{a}^{66}$ ! - il se rend près d'une fenêtre et lit son texte mot à mot ${ }^{67}$.

Son déjeuner est pris au milieu de l'après-midi ou presque, puisqu'il ne manque pas de jaser avec tout le monde une fois la messe finie. Pas tellement longtemps après cela, lui et ses gens sont de retour à l'église

63 Témoignages de $M^{\text {me }}$ Everet Chevraux et de $M$. et $M^{\text {me }}$ John Martz donnés à E. O. Drouin en 1952.

64 Témoignage de $\mathrm{M}^{\mathrm{me}}$ Fred Dittberner donné à E. O. Drouin en 1952.

65 LL, HB à JMB, 3-1-1883, 7-1-1883, 18-1-1884, 15-1-1888; L, HB à une demoiselle de France, 26-2-1888; témoignage de $M$. et $\mathrm{M}^{\mathrm{me}}$ John Martz et témoignage de $M^{\text {me }}$ Fred Dittberner donnés à E. O. Drouin en 1952.

66 Témoignage d'Alexander Ross donné à E. O. Drouin en 1952.

67 Témoignage de $\mathbf{M}^{\mathrm{me}}$ Fred Dittberner donné à E. O. Drouin en 1952. 
pour la bénédiction du Très Saint Sacrement et même parfois pour les Vêpres ${ }^{68}$.

La cérémonie qui a le plus de retentissement dans le pays est bien la procession de la Fête-Dieu d'abord en 1808 puis ensuite tous les ans sans faillir ${ }^{69}$. Elle est annoncée d'avance, de sorte qu'un grand concours de gens d'un peu partout s'y rendent. A environ un quart de mille sur le haut de la vallée, dans une épinettière, on construit une haute estrade où est placé l'autel du reposoir. A l'aller et au retour, les chants enthousiastes de motets et de cantiques par toute la foule sont ponctués de décharges de mousqueterie. Des jeunes gens et des jeunes filles transportent quelques statues ou tiennent en main de nombreuses bannières et des fanions. Durant plusieurs années les missionnaires d'Hobbéma y viennent en compagnie de religieuses et d'un orchestre de mandolines eniraîné par le père Pierre Moulin.

En souvenir des Beillevaire et peut-être un peu par nostalgie des temps jadis, les gens, chaque année, tiennent à ce que cette procession ait lieu à partir de l'ancienne église, quoiqu'elle soit désaffectée et qu'elle soit devenue un musée.

Il faut souligner enfin les retraites ou missions paroissiales. Dès 1884, le père Scollen prêche la première ${ }^{70}$. Elles se répètent à intervalles plus ou moins rapprochés par la suite. Par exemple, en 1900, ce sera le tour d'un Oblat métis du nom de Cunningham, originaire de Saint-Albert, de s'y rendre dans ce but ${ }^{71}$. Enfin, deux documents ${ }^{72}$ mentionnent une retraite annuelle depuis 1929 à 1936 inclusivement.

Pastorale de chrétienté, pastorale d'évangélisation, il est certain que Beillevaire, tout comme les autres missionnaires des prairies, ne se dérange pas pour en connaître les définitions des théoriciens. Ils font de leur mieux dans des circonstances souvent adverses et toujours difficiles. Ils sont sans cesse mêlés à tout ce qui regarde les individus, les familles et les groupes sous leur juridiction parce que cela est nécessaire, mais ils visent, bien entendu, à faire de vrais chrétiens de leurs gens. Évidemment, la réussite ne répond pas, bien souvent, à leurs désirs et à leur prière mais, en tout cas, ils font leur possible et c'est tout ce que la Providence peut leur demander.

Éméric Drouin, o.m.i.. B.Éd.. M.Éd.. Ph.D., Curé de Sainte-Famille de Calgary, Alherta.

68 L, HB à JMB, 30-1-1895.

69 L, HB à une demoiselle de France, 26-2-1888; LL, HB à JMB, 27-1]-1895, 2-6-1905, 1905, 28-7-1905; LL, HB à M, 14-6-1907, 21-6-1921; LL, B à HB, 29-7-1926, 24-6-1933; témoignage d'Alexander Ross donné à E. O. Drouin en 1952.

$70 \mathrm{~L}$ du père Leduc, o.m.i., à Les Missions ...., livraison de mars 1885, \# 89.

$71 \mathrm{M}^{\mathrm{gr}}$ Emile Legal, Journal quotidien, 16-3-1900.

72 L, HB à B, 17-6-1929; L, B. W. Malone, c.s.s.r., à HB, 11-7-1935. 